\title{
Light collection engines with micro compound-eye arrays for pico-projection displays
}

\author{
Heng ZHAO*, Jun WANG, Qing YAN, and Deng-xin HUA \\ The School of Mechanical and Precision Instrument Engineering of Xi'an University of Technology, \\ 5 South Jinhua Road, Xi'an, Shaanxi, PR China \\ *Corresponding author: hzhao@xaut.edu.cn
}

Keywords: Pico-projection display, Compound-eye array, Compound parabolic concentrator.

\begin{abstract}
Light emitting diodes (LEDs) have recently gained much interest as projection light sources due to their long lifetimes, large color gamut, small size, and absence of mercury vapor. In this work, a compound parabolic concentrator (CPC) coupled to a compound-eye array is designed and fabricated as a light collection engine of LED-based pico-projection diaplay. The results indicate that more than $90 \%$ light can be collected by the CPC coupled to a compound-eye array and transmitted within the designed angle. This method is advantageous in many respects compared with those available, such as compact volume, high collection efficiency, rectangular radiation pattern and controllable output divergence angle. These features and results validate the potential of a CPC coupled to a compound-eye array as a light collection engine of pico-projection display application.
\end{abstract}

\section{Introduction}

Regarded as the fourth generation light sources, light-emitting-diode-based lighting devices have gradually become alternative light sources in projection display application. Many new small format projectors are switching out high intensity discharge lamps for discrete LEDs, resulting in smaller form factors and enabling pico-projection technologies. Representing an entirely new projector category, pico-projectors are sharing many mature technologies with their "big brothers". However, there is a bottleneck that the luminous output of LEDs is still lower than that of UHP lamps in pico-projection display. Therefore, it is important to improve light utilization efficiency of LEDs for pico-projection display application [1].

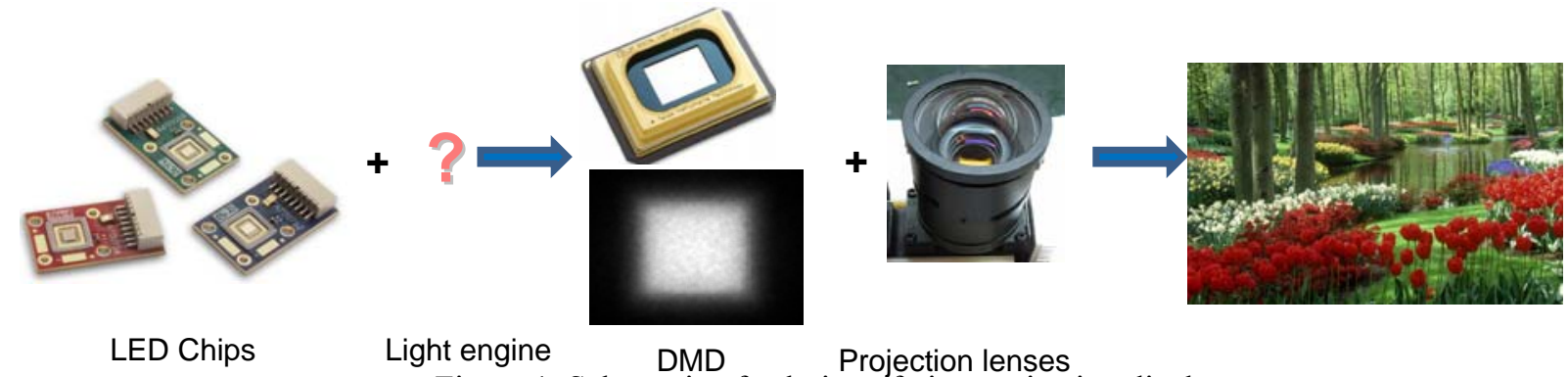

Figure 1. Schematic of solution of pico-projection display.

A pico-projector consists of three parts: light source, light engine, projection lens, as shown in Fig. 1. More important, at the heart of all projectors is the light engine. Traditionally, an LED die is encapsulated in dome lens. Its radiation pattern is circular. Since the panel of the pico-projector is rectangular and the projection system is an étendue-limited system, the light beam emitting from the LED should be collimated and homogenized by the light engine, which transforms the circular radiation pattern to rectangular radiation pattern within a certain acceptance angle. In this process, however, the light utilization efficiency of the LED is low, so a new solution is needed to enhance the light utilization efficiency. This étendue issue is identical to the étendue issue that drove LED-based projectors to use the shortest possible arc with the highest possible are luminance [2]. Optical improvements allow more of the light extracted from the die to be focused on the digital micro-mirror device (DMD). 
In this work, a compound parabolic concentrator (CPC) coupled to a compound-eye array is designed and fabricated as a light collection engine of LED-based pico-projection diaplay. One of the great advantages of this configuration is its great energy efficiency. There is no auxiliary focusing lens required in this configuration, another advantage which simplifies the overall system architecture. Often the functions of light transport and integration are combined [3]. In addition, by tailoring the diffuser properties, the output étendue may be tailored to match the microdisplay to be used.

\section{Methods}

\section{1 Étendue}

Étendue is one of the most basic yet important concepts in the design of projection display. First, it explains the flux transfer characteristics of the optical system. Second, it plays an integral role in the ability to shape the distribution of radiation at the target [4].

The definition of étendue $\zeta$ is

$$
\zeta=n^{2} \iint \cos \theta d A d \Omega
$$

where $\mathrm{n}$ is the index of refraction in source space, $\mathrm{dA}$ is the area of the interest, $\theta$ is the angle between the center of the emission cone and the normal to the emitting area. In addition, $d \Omega$ is the solid angle of the light corresponding to that area.

To this point, the étendue has been described as a property of an optical system. However, the definition can be extended to consider the étendue of a source. The source étendue describes the geometric flux emission characteristics of the emitter by itself, and it provides a fundamental limit on the ability of a following optical system to collect and transmit this flux to the intended target.

A projection system is referred to as "étendue-limited" when there is an optical element or combination of optical element in the system whose étendue is lower than that of the ray bundle produced by the source/reflector combination. Most practical étendue-limited projection systems are limited by the étendue of microdisplay. According to Eq. 1, the étendue of the microdisplay $\zeta_{\text {md }}$ could be written as

$$
\zeta_{\mathrm{md}}=\pi A(N . A .)^{2}
$$

where N.A. is the numerical aperture of the projection lens, and A is the panel area of the microdisplay. Note that the étendue is a geometric quantity that describes the flux propagation characteristics for a lossless system. The term lossless puts constraints on the interpretation of a system étendue.

\subsection{Design of a CPC}

In this work, a performance of a 0.8 inch panel and a projection lens with a numerical aperture of 0.2 is made. Meanwhile, a $2 \mathrm{~mm} \times 2 \mathrm{~mm}$ monolithic LED chip with an area of $6.5 \mathrm{~mm}^{2}$ is implemented as a pico-projection source in a simple optical setup. The incident angle of rays is required as about $12^{\circ}$ for the DMD device of microdisplay. By Eq. 1, the étendue of LED source is $20.3 \mathrm{~mm}^{2} \bullet \mathrm{sr}$. And by Eq. 2, the étendue of microdisplay is $26.3 \mathrm{~mm}^{2} \cdot \mathrm{sr}$.
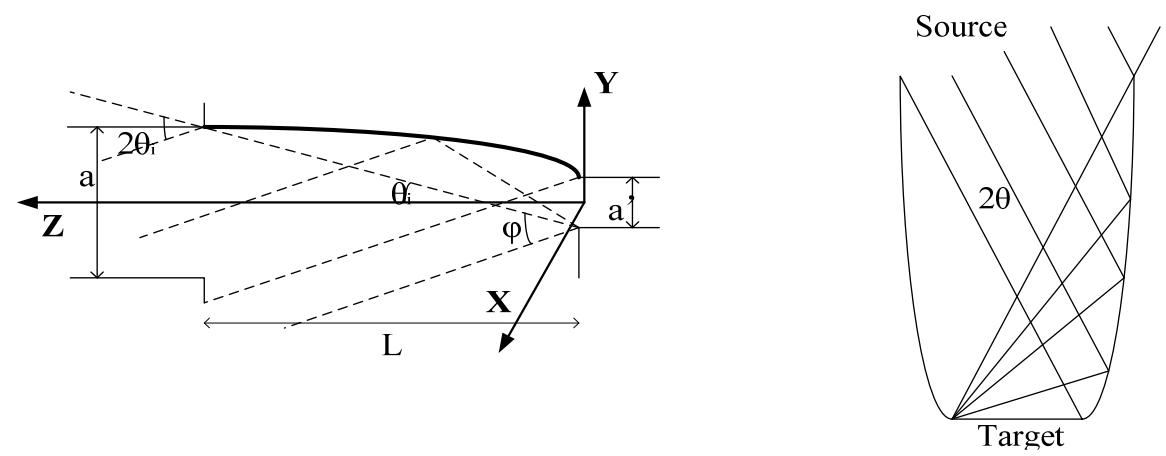

Figure 2. Schematic of CPC. 
According to the edge-ray principle of non-imaging optics and the theory of étendue, a proper design of a CPC is given. The angle $\theta_{i}$ with which light rays reach the exit aperture of the CPC is given by

$$
\sin \theta_{i}=a^{\prime} / a
$$

where $\mathrm{a}$ is the semi-diameter of the entrance aperture, and a is the semi-diameter of the exit aperture, as shown in Fig. 2.

The focal length of the parabolic curve is

$f=a^{\prime} /\left(1+\sin \theta_{i}\right)$

The length of the CPC is given by

$L=(a+a) / \tan \theta_{i}$

In this work, a monolithic LED chip is encapsulated in the bottom recess of the CPC. The recess is full of silicone with a refractive index of 1.5. If LED dies are encapsulated in the silicone with a refractive index of 1.5, the étendue of LED will be increased by a factor of 2.2. However, the extraction efficiency of a LED in encapsulation is 2.3 times higher than that in air. Therefore, the encapsulation solution results in less of a heat dissipation problem that can reduce LED output.

2.3 Design of a compound-eye array

An illumination-uniformity study can be performed using a radiometric analysis by considering each LED on a monolithic LED array as a non-perfect Lambertian and incoherent emitter. In practice, this dependence turns out to be a power law that mostly depends on the encapsulant and semiconductor region shapes. A practical approximation for the irradiance distribution over the compound-eye array is given by

$$
E(r, \theta)=E_{0}(r) \cos ^{m} \theta
$$

where $\theta$ is the viewing angle and $E$ is the irradiance on axis at distance $r$ from the LED chip. The value of $m$ depends on the relative position of the LED chip emitting region from the curvature center of the parabolic curve of CPC.

The irradiance distribution given by Eq. 6 for a LED displaced to position $\left(\mathrm{x}_{0}, \mathrm{y}_{0}\right)$ over a compound-eye array can be written in terms of Cartesian coordinates $(\mathrm{x}, \mathrm{y}, \mathrm{z})$. The irradiance at a point $\mathrm{P}(\mathrm{x}, \mathrm{y}, \mathrm{z})$ then is expressed as

$$
E(x, y, z)=\frac{z^{m} L A}{\left[\left(x-x_{0}\right)^{2}+\left(y-y_{0}\right)^{2}+z^{2}\right]^{(m+2) / 2}}
$$

where $L$ is the radiance of the LED source and $A$ is the area of a compound-eye array.

In terms of assembly requirements, the most popular extended compound-eye array is the square. For a square array, the irradiance $E$ is given by the sum of the irradiances of a matrix of $N \times M$ compound eyes, as shown in Fig. 3.

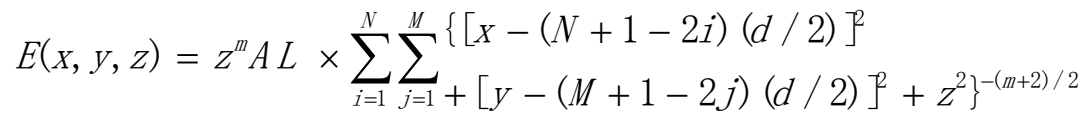

$$
\begin{aligned}
& 0000000 \\
& 0000000 \\
& 00000000 \\
& 0000000
\end{aligned}
$$

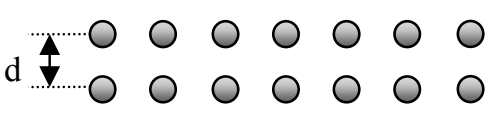

$$
\begin{aligned}
& \stackrel{0}{\leftrightarrow} 000000 \\
& \text { d }
\end{aligned}
$$

Figure 3. Schematic of square compound-eye array with d spacing. 


\subsection{A CPC coupled to a compound-eye array}

A light collection engine consists of several parts: a monolithic LED array, a rectangular CPC and a compound-eye array [5]. The PMMA is used as the material of the CPC coupled to a compound-eye array. The profile of the light collection engine by ProE® is shown in Fig. 4(a).

The bottom recess of the rectangular CPC is in the shape of cuboid with a length of $3.2 \mathrm{~mm}$, a width of $2.5 \mathrm{~mm}$ and a height of $0.5 \mathrm{~mm}$. LED dies are encapsulated by the silicone in the recess. From the N.A. of the pico-projector lens, the maximum value of $\theta$ is $12^{\circ}$. By Eq. 6 , the length of the CPC is given as $35.3 \mathrm{~mm}$, as shown in Fig. 4(b).

The next step is to determine the structure of the compound-eye array, which is coupled together in the top of the CPC. The aperture and focal length of compound eye lens are determined according to the size of rectangular CPC and the aperture angle of lighting systems. The diameter of the output aperture is $20.87 \mathrm{~mm}$ and the length of the CPC is $59.31 \mathrm{~mm}$. Upon making use of Eq. 7 - 8, a $125 \times 125$ micro array is obtained as the structure of compound-eye with $80 \mu \mathrm{m}$ in diameter.

All components of the optical system are held inside a Polymethylmethacrylate (PMMA) cylinder to make the system mechanically feasible. The LED chip is put at the bottom of the CPC with a distance of $0.1 \mathrm{~mm}$, and the entrance area of the CPC covers the monolithic LED chip completely. Meanwhile, the compound-eye array is set at the top of the CPC, as shown in Fig. 4(c). Eventually, the ray tracing results are shown in Fig. 4(d).

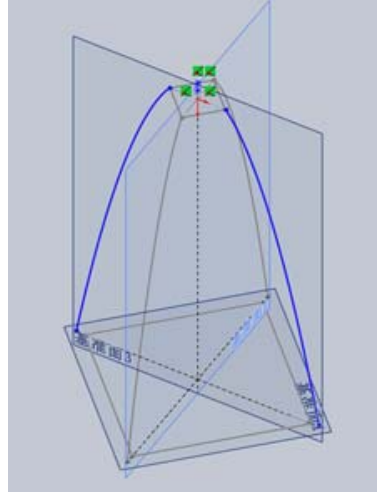

(a)

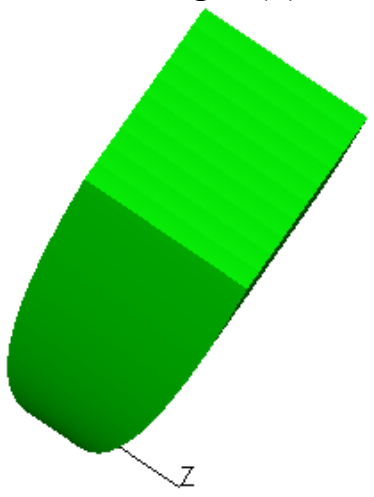

(b)

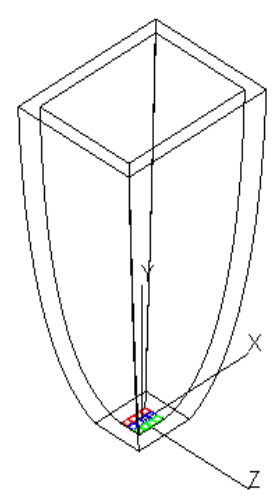

(c)

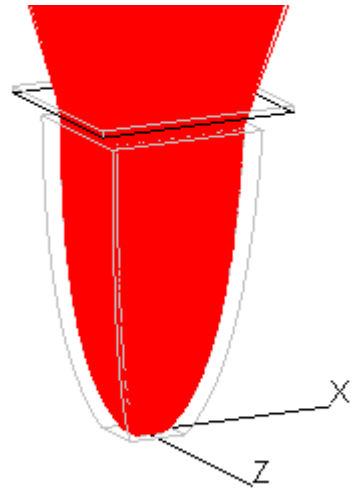

(d)

Figure 4. Schematic of light collection engine. (a) Profile of light collection engine by ProE®. (b) 3D rendering of light collection engine by ProE®. (c) Wireframe of light collection engine imported into TracePro®. (d) Raytrace of light collection engine by TracePro $\AA$.

Simulation results are shown in Fig. 5. It is noted that the light collection engine has a high collection efficiency of $90.5 \%$. The uniformity is also an important parameter in projection display application. Fig. 5(a) pictures the simulated uniformity of the light collection engine. It is found that variations of the exit light intensity across the target surface are typically minor based on the coupled structural design. In addition, it should be noted that the light collection engine has the capacity that the intensity is uniform across distribution angle from $-12^{\circ}$ to $12^{\circ}$. Then, the intensity gradually falls off, as shown in Fig. 5(b). This result shows that the system meets the design requirement.

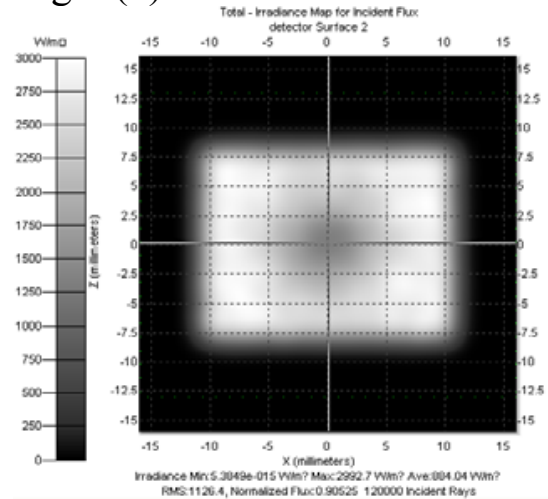

(a)

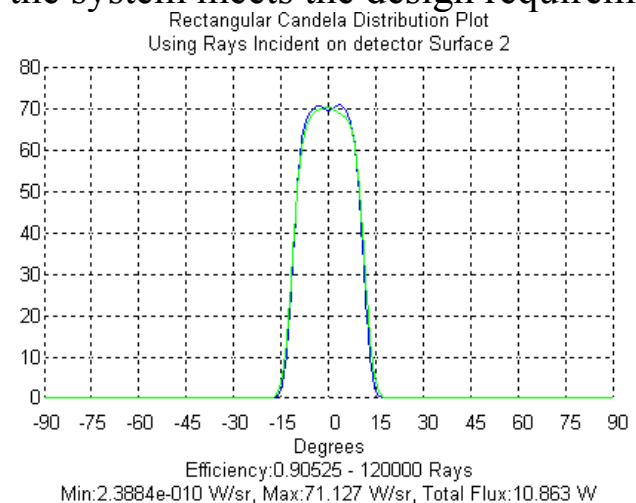

(b)

Figure 5. Simulated result. (a) Uniformity of exit light intensity distribution for light collection engine. (b) Candela distribution for light collection engine. 


\section{Experimental}

According to the simulation results, the light collection engine is fabricated as followed. The production process of the compound-eye array is first briefly introduced, which is a two-step process of mold with concave microstructures and inject molding of polymer convex microlenses [6].

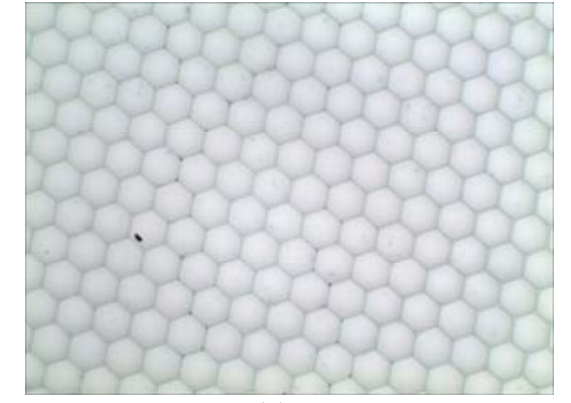

(a)

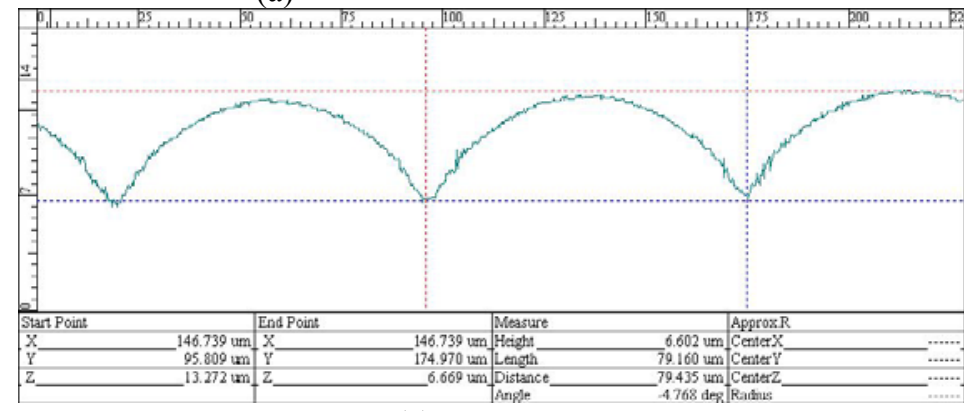

(c)

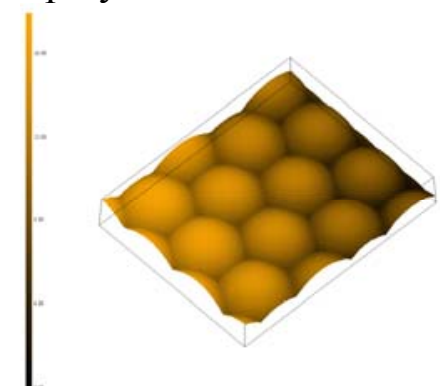

(b)

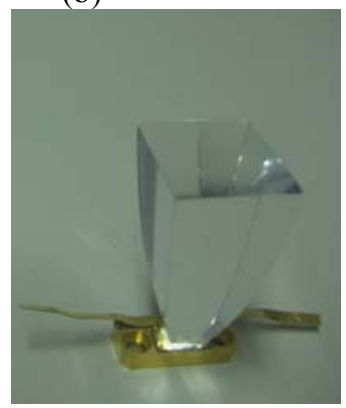

(d)

Figure 6. Actual sample of light collection engine. (a) SEM image of compound-eye array. (b) 3D profile of array which is captured by 3D laser confocal microscope. (c) Cross-section profile and the measured results of compound-eye array. Average height of compound eyes is $6.602 \mu \mathrm{m}$, and width is $79.160 \mu \mathrm{m}$. (d) Photograph of actual sample.

Step 1: Acid-assisted rapid manufacture of the mold. The glass mold of the array with rectangular shape is prepared in this section. First, a femtosecond laser beam is carried out point-by-point on a polished silica glass chip $\left(11 \times 11 \mathrm{~mm}^{2}\right)$ with a 30 -fs and $800-\mathrm{nm}$ laser pulses at a repetition of $1 \mathrm{kHz}$. Second, the sample with craters is treated in hydrofluoric acid solution assisted by an ultrasonic bath at about $23^{\circ} \mathrm{C}$. During this process, the chemical etch is significantly enhanced in the laser-induced craters, and the concave spherical surfaces begin to form in the laser-exposure spots [7]. The circular-shaped concave structures expand with the chemical etching process. When the neighbor ones contact with each other, straight boundary lines are formed, eventually forming the hexagonal patterns, as shown in Fig. 6(a).

Step 2: Inject molding. For replication of micro-structures, the PMMA sheet and mold were fixed onto upper and lower stages of the system. The glass mold is heated to $120^{\circ} \mathrm{C}$ by an electric heating template with a power of $400 \mathrm{~W}$. The PMMA sheet is moved downwards and pressed on the heated mold. As the heating process terminates, the temperature declines gradually. At the temperature below $50^{\circ} \mathrm{C}$, the process of stripping PMMA sheet is made to form the compound-eye array. Figs. 6(b) and (c) show the SEM images of the mold, a fact which demonstrates that the uniform and smooth surface can be made by this method.

Step 3: Bond molding. According to the design of CPC earlier, the dielectric rectangular CPC is fabricated with PMMA by injection molding, extrusion and casting processing. Then, it bonds well with the compound-eye array previously prepared by the thermal pressed treatment. Finally, the light collection engine comes into shape with the structure of the dielectric rectangular CPC coupled to a compound-eye array, as shown in Fig. 6(d).

The performance of red LED module is then tested and characterized. There is shown a radiation pattern of integrated with red LED modules under power-on test in Fig. 7(a). It is found that the output radiation pattern has been transformed to the rectangular pattern which is matched well with the microdisplay panel. The relative intensity distribution of the LED module is shown in Fig. 7(b). It is noted that the light beam emitting from the LED chip has been collimated effectively. The 
divergence angle of the light beam exiting the LED module is $13^{\circ}$, which is consistent with the computer emulation.

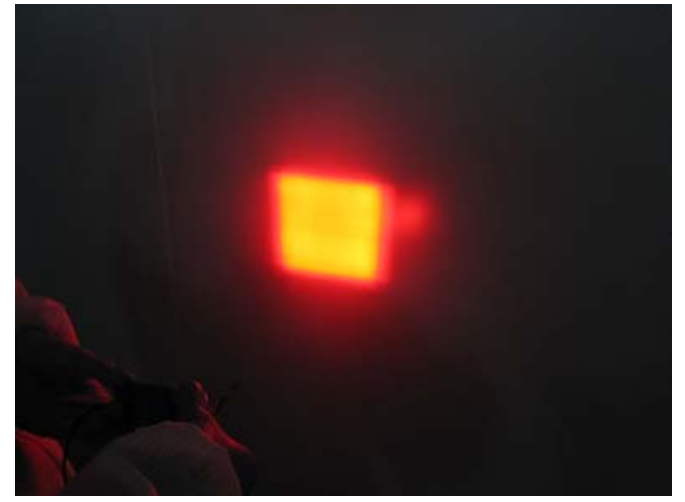

(a)

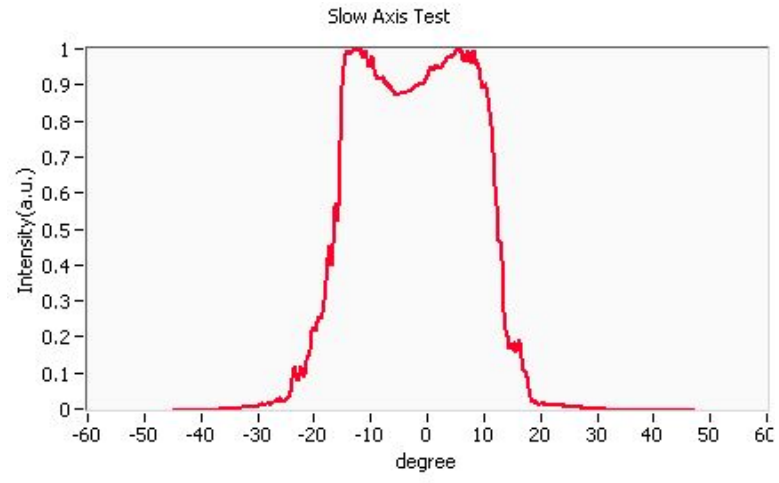

(b)

Figure 7. Prototype test on the light collection engine packaged a red LED module. (a) The pictures of the light patterns for the demo. (b) Exit light intensity distribution with varying light output angle.

\section{Conclusion}

In summary, a coupled structure is presented for a light collection engine. A rectangular dielectric CPC coupled to a compound-eye array is designed and optimized to improve the light utilization efficiency for pico-projector application. More than 90\% light emitted by the LED array can be collected by this CPC and transmitted within the designed angle. This method is advantageous in many respects compared with those available, such as compact volume, high collection efficiency, rectangular radiation pattern and controllable output divergence angle.

Moreover, the microstructures with a compound-eye array are created in one mold in this work, and the shapes are simply controlled individually by rapid prototyping technology based on femtosecond laser pulses. This method provides an alternating approach for the fast fabrication of large-area polymeric micro-optical devices with various shapes in further study.

\section{Acknowledgement}

The authors would like to acknowledge support from the Scientific Research Programs of Shaanxi Provincial Department of Education grant 12JK0660, as well as the Natural Science Foundation of Shaanxi Provincial Department of Science and Technology grant 2013JQ7002.

\section{References}

[1] H. Hank, For MEMS Displays, Projections of Success, Photonics Spectra. 50 (2016) 36-40.

[2] Y. H. Ra; R. Wang; S. Y. Woo, Full-Color Single Nanowire Pixels for Projection Displays, Nano Letters. 16 (2016) 4608-4615.

[3] M. Gajic; N. Karwa; A. Mojiri, Modeling reflection loss from an evacuated tube inside a compound parabolic concentrator with a cylindrical receiver, Optics Express. 23 (2015) A493-A501.

[4] R.J. Koshel, Illumination engineering: design with nonimaging optics, in: J. Koshel (Eds.), Etendue, John Wiley \& Sons Inc., New York, 2013, pp. 31-40.

[5] M. William; G. M. Schuster; J. E. Ford, Planar waveguide LED illuminator with controlled directionality and divergence, Optics Express. 22 (2014) A742-A758.

[6] K. H. Jeong, J. Kim, L. P. Lee, Biologically inspired artificial compound eyes, Science. 312 (2006) 557-561.

[7] C. C. Huang; X. Wu; H. Liu, Large-Field-of-View Wide-Spectrum Artificial Reflecting Superposition Compound Eyes, Small. 10 (2014) 3050-3057. 